\title{
Norm inequalities for higher-order commutators of one-sided oscillatory singular integrals
}

\section{Shaoguang Shi* and Lei Zhang}

\section{"Correspondence:}

shishaoguang@lyu.edu.cn

Department of Mathematics, Linyi

University, Linyi, 276005, P.R. China

\begin{abstract}
In the present paper, we study the weighted norm inequalities for higher-order commutators formed by a class of one-sided oscillatory singular integrals and BMO functions. We obtain that the boundedness of these commutators can be deduced by that of one-sided Calderón-Zygmund singular integral operators.
\end{abstract}

MSC: Primary 42B20; secondary 42B25

Keywords: commutator; one-sided oscillatory integral; one-sided weight

\section{Introduction}

The aim of this paper is to further study the one-sided version of the following oscillatory singular integral, which was first introduced and studied by Ricci and Stein [1]:

$$
T f(x)=\text { p.v. } \int_{\mathbb{R}^{n}} e^{i P(x, y)} K(x-y) f(y) d y,
$$

where $P(x, y)$ is a nontrivial real-valued polynomial defined on $\mathbb{R}^{n} \times \mathbb{R}^{n}$, and $K$ is a Calderón-Zygmund kernel. We say that a function in $L_{\text {loc }}^{1}\left(\mathbb{R}^{n} \backslash\{0\}\right)$ is a Calderón-Zygmund kernel if the following properties are satisfied [2]:

(1) there exists a finite constant $C_{1}$ such that

$$
|K(x-y)-K(x)| \leq \frac{C_{1}|y|}{|x|^{2}} \quad \text { for all }|x|>2|y|
$$

(2) there exists a finite constant $C_{2}$ such that

$$
\left|\int_{\varepsilon<|x|<N} K(x) d x\right| \leq C_{2} \quad \text { for all } \varepsilon \text { and } N \text { such that } 0<\varepsilon<N
$$

(3) there exists a finite constant $C_{3}$ such that

$$
|K(x)| \leq \frac{C_{3}}{|x|} \quad \text { for all } x \neq 0 .
$$

(c) 2016 Shi and Zhang. This article is distributed under the terms of the Creative Commons Attribution 4.0 International License (http://creativecommons.org/licenses/by/4.0/), which permits unrestricted use, distribution, and reproduction in any medium, provided you give appropriate credit to the original author(s) and the source, provide a link to the Creative Commons license, and indicate if changes were made. 
Ricci and Stein [1] studied the norm inequalities for $T$ on $L^{p}\left(\mathbb{R}^{n}\right)$ spaces with $1<p<\infty$. Weighted inequalities arise naturally in Fourier analysis, but their use is best justified by the variety of applications in which they appear. For example, the theory of weights plays an important role in the study of boundary value problems inherent in Laplace equations on Lipschitz domains. Many people are interested in the study of the events that occur when the weight function belongs to the Muckenhoupt classes $A_{p}$ :

$$
\left(\frac{1}{|B|} \int_{B} w(x) d x\right)\left(\frac{1}{|B|} \int_{B} w(x)^{1-p^{\prime}} d x\right)^{p-1} \leq C
$$

Here $1<p<\infty$, and $B$ denotes any ball in $\mathbb{R}^{n}$. The classes $A_{1}$ are defined as $M w \leq C w$, where $M$ is the classical Hardy-Littlewood maximal operator. Lu and Zhang [3, 4] gave the boundedness of $T$ on $L^{p}(w)(1<p<\infty)$ spaces with weight functions $w \in A_{p}$. For other classical works on $T$, see, for example, [5-10] and the references therein. In what follows, we restrict our attention on $n=1$ in order to introduce the one-sided operators defined on $\mathbb{R}$.

Many operators in harmonic analysis have one-sided versions. It is well known that the one-sided Hardy-Littlewood maximal operators are required in ergodic theory. Sawyer [11] introduced the integral version of these operators as

$$
M^{+} f(x)=\sup _{h>0} \frac{1}{h} \int_{x}^{x+h}|f(y)| d y \text { and } M^{-} f(x)=\sup _{h>0} \frac{1}{h} \int_{x-h}^{x}|f(y)| d y \text {. }
$$

The good weights for $M^{+}$and $M^{-}$are the one-sided version of $A_{p}$ classes. Sawyer [11] studied these one-sided weights in depth for the first time. We recall their definitions:

$$
\begin{aligned}
& A_{p}^{+}: \sup _{a<b<c} \frac{1}{(c-a)^{p}} \int_{a}^{b} w(x) d x\left(\int_{b}^{c} w(x)^{1-p^{\prime}} d x\right)^{p-1} \leq C \text { for } 1<p<\infty, \\
& A_{p}^{-}: \sup _{a<b<c} \frac{1}{(c-a)^{p}} \int_{b}^{c} w(x) d x\left(\int_{a}^{b} w(x)^{1-p^{\prime}} d x\right)^{p-1} \leq C \quad \text { for } 1<p<\infty,
\end{aligned}
$$

and

$$
A_{1}^{+}: M^{-} w \leq C w \text { and } A_{1}^{-}: M^{+} w \leq C w .
$$

In [12], the classes $A_{\infty}^{+}$and $A_{\infty}^{-}$were introduced as

$$
A_{\infty}^{+}=\bigcup_{1 \leq p<\infty} A_{p}^{+} \quad \text { and } \quad A_{\infty}^{-}=\bigcup_{1 \leq p<\infty} A_{p}^{-} .
$$

The important point to note here is that the one-sided Muckenhoupt classes are larger than the classical Muckenhoupt classes. For instance, the function $e^{x} \in A_{1}^{+}$, but $e^{x} \notin A_{1}$. In fact, it is easy to see that for $1 \leq p \leq \infty, A_{p} \subsetneq A_{p}^{+}, A_{p} \subsetneq A_{p}^{-}$, and $A_{p}=A_{p}^{+} \cap A_{p}^{-}$. Furthermore, both the reverse Hölder inequality and the doubling condition are not true for the onesided Muckenhoupt classes. Therefore, some different ideas are needed here to deal with the weighted norm inequalities for one-sided operators. The classes $w \in A_{p}^{+}$are of interest, 
not only because they control the boundedness of the one-sided Hardy-Littlewood maximal operators, but also they are the right classes for the weighted estimates of one-sided Calderón-Zygmund singular integral operators [13] defined by

$$
\widetilde{T}^{+} f(x)=\lim _{\varepsilon \rightarrow 0^{+}} \int_{x+\varepsilon}^{\infty} K(x-y) f(y) d y \quad \text { and } \quad \widetilde{T}^{-} f(x)=\lim _{\varepsilon \rightarrow 0^{+}} \int_{-\infty}^{x-\varepsilon} K(x-y) f(y) d y,
$$

where $K$ is the Calderón-Zygmund kernel with support in $\mathbb{R}^{-}=(-\infty, 0)$ and $\mathbb{R}^{+}=(0,+\infty)$, respectively (also called the one-sided Calderón-Zygmund kernel). An example of such a kernel is

$$
K(x)=\frac{\sin (\log |x|)}{(x \log |x|)} \chi_{(-\infty, 0)}(x)
$$

where $\chi_{E}$ denotes the characteristic function of a set $E$.

Highly inspired by these statements for the oscillatory singular integral operators and the one-sided operator theory, $\mathrm{Fu}, \mathrm{Lu}, \mathrm{Shi}$, and their coauthors introduced the one-sided oscillatory singular integral operators and studied some weighted norm inequalities for these operators with one-sided weights, including the strong weighted $L^{p}(1<p<\infty)$ boundedness [14], the weighted weak $(1,1)$ type norm inequalities [15] and the weighted norm estimates on one-sided Hardy spaces [16]. We recall the definition of one-sided oscillatory integral operators:

$$
T^{+} f(x)=\lim _{\varepsilon \rightarrow 0^{+}} \int_{x+\varepsilon}^{\infty} e^{i P(x, y)} K(x-y) f(y) d y
$$

and

$$
T^{-} f(x)=\lim _{\varepsilon \rightarrow 0^{+}} \int_{-\infty}^{x-\varepsilon} e^{i P(x, y)} K(x-y) f(y) d y,
$$

where $P(x, y)$ are real-valued polynomials defined on $\mathbb{R} \times \mathbb{R}$, and $K$ are the one-sided Calderón-Zygmund kernels.

Let $b$ be a locally integrable function on $\mathbb{R}^{n}$, and let $\mathcal{T}$ be an integral operator. Then we define the commutator operator for a proper function $f$ by

$$
\mathcal{T}_{b}(f):=b(\mathcal{T} f)-\mathcal{T}(b f)
$$

The function $b$ is also called the symbol function of $\mathcal{T}_{b}$. The investigation of the operator $\mathcal{T}_{b}$ begins with Coifman-Rochberg-Weiss pioneering study of the operator $\mathcal{T}$ [17]. There are two major reasons for considering the problem of commutators. The first one is that the boundedness of commutators can produce some characterizations of function spaces $[18,19]$. The other one is that the theory of commutators plays an important role in the study of the regularity of solutions to elliptic and parabolic partial differential equations (PDEs) of the second order [20,21]. It is well known that many people are interested in the study of commutators for which the symbol functions belong to $B M O$ spaces. In harmonic analysis, a function $b$ of bounded mean oscillation, also known as a $B M O$ function, is a real-valued function whose mean oscillation is bounded, that is,

$$
\frac{1}{|B|} \int_{B}\left|b(x)-b_{B}\right| d x<\infty
$$


where $B$ is a ball in $\mathbb{R}^{n}$. The $B M O$ space is the function space with the norm

$$
\|b\|_{B M O\left(\mathbb{R}^{n}\right)}=\sup _{B} \frac{1}{|B|} \int_{B}\left|b(x)-b_{B}\right| d x .
$$

In some precise sense, the space $B M O\left(\mathbb{R}^{n}\right)$ plays the same role in the theory of Hardy spaces $H^{p}(0<p<1)$ as that the space $L^{\infty}$ of essentially bounded functions plays in the theory of $L^{p}$ spaces with $1<p<\infty$. The commutator formed by the oscillatory integral operator $T$ and a $B M O$ function $b$ can be defined by

$$
T_{b} f(x)=\text { p.v. } \int_{\mathbb{R}^{n}} e^{i P(x, y)} K(x-y)(b(x)-b(y)) f(y) d y .
$$

The commutators of degree $k\left(k \in \mathbb{Z}^{+}\right)$of $T$ were defined by

$$
T_{b}^{k} f(x)=\text { p.v. } \int_{\mathbb{R}^{n}} e^{i P(x, y)} K(x-y)(b(x)-b(y))^{k} f(y) d y .
$$

It is immediate that $T_{b}^{0}=T$. In the literature, there are a great deal of results on the norm inequalities for $T_{b}^{k}$; see, for example, [22-28] and the references therein.

For the one-sided case, Lorente and Riveros $[29,30]$ introduced the commutators of some one-sided operators. Here we collect the definitions of commutators for the onesided Hardy-Littlewood maximal operators and the one-sided Calderón-Zygmund singular integral operators as follows. Let $k=0,1, \ldots$ and $b \in B M O(\mathbb{R})$. The commutators of degree $k$ generated by $M^{+}\left(M^{-}\right)$and $b$ are defined by

$$
M_{b}^{k,+} f(x)=\sup _{h>0} \frac{1}{h} \int_{x}^{x+h}|b(x)-b(y)|^{k}|f(y)| d y
$$

and

$$
M_{b}^{k,-} f(x)=\sup _{h>0} \frac{1}{h} \int_{x-h}^{x}|b(x)-b(y)|^{k}|f(y)| d y .
$$

The $k$ th commutators generated by $\widetilde{T}^{+}\left(\widetilde{T}^{-}\right)$and $b$ are defined by

$$
\widetilde{T}_{b}^{+, k} f(x)=\text { p.v. } \int_{x}^{\infty} K(x-y)(b(x)-b(y))^{k} f(y) d y
$$

and

$$
\widetilde{T}_{b}^{-, k} f(x)=\text { p.v. } \int_{-\infty}^{x} K(x-y)(b(x)-b(y))^{k} f(y) d y .
$$

Here $K$ are one-sided Calderón-Zygmund kernels defined as before. The corresponding weighted norm inequalities for these commutators will be stated in Section 2. Highly inspired by the results for one-sided operators, Fu et al. [31] introduced the commutators formed by $T^{+}\left(T^{-}\right)$and $b \in B M O(\mathbb{R})$ as follows:

$$
T_{b}^{+} f(x)=\text { p.v. } \int_{x}^{\infty} e^{i P(x, y)} K(x-y)(b(x)-b(y)) f(y) d y
$$


and

$$
T_{b}^{-} f(x)=\text { p.v. } \int_{-\infty}^{x} e^{i P(x, y)} K(x-y)(b(x)-b(y)) f(y) d y .
$$

For $k \in \mathbb{Z}^{+}$, the high-order commutators of $T^{+}\left(T^{-}\right)$and $b \in B M O(\mathbb{R})$ can be deduced as

$$
T_{b}^{k,+} f(x)=\text { p.v. } \int_{x}^{\infty} e^{i P(x, y)} K(x-y)(b(x)-b(y))^{k} f(y) d y
$$

and

$$
T_{b}^{k,-} f(x)=\text { p.v. } \int_{-\infty}^{x} e^{i P(x, y)} K(x-y)(b(x)-b(y))^{k} f(y) d y .
$$

This paper is devoted to the weighted norm inequalities for $T_{b}^{k,+}$ and $T_{b}^{k,-}$ with $k>1$. Due to their similarities, we further consider only the operator $T_{b}^{k,+}$.

Now, we can formulate our results.

Theorem 1.1 Let $1<p<\infty, k \in \mathbb{Z}^{+}, w \in A_{p}^{+}$, and let $b \in B M O(\mathbb{R})$. Then the operator $T_{b}^{k,+}$ is bounded on $L^{p}(w)$.

Theorem 1.2 Let $p, w$, and $b$ be defined as in Theorem 1.1. Then the truncated operator

$$
\widetilde{T}_{b, 0}^{k,+} f(x)=\text { p.v. } \int_{x}^{x+1} K(x-y)(b(x)-b(y))^{k} f(y) d y
$$

is bounded on $L^{p}(w)$.

We end this section with the outline of this paper. In Section 2, some lemmas are collected for the proofs of our main results. Section 3 contains the proofs of Theorem 1.1 and Theorem 1.2. Throughout this paper, the letter $C$ will denote a positive constant that may vary from line to line but will remain independent of the relevant quantities.

\section{Basic lemmas}

We provide in this section some lemmas that are crucial for the proofs in Section 3. Together with the characterizations of the weighted inequalities for one-sided operators, we can obtain some properties of the classes $A_{p}^{+}$and $A_{p}^{-}$.

Lemma $2.1[11,14,32]$

(1) If $w \in A_{p}^{+}$, then $w^{1+\varepsilon} \in A_{p}^{+}$for some $\varepsilon>0$ with $1 \leq p<\infty$.

(2) Let $1<p<\infty$. Then $w \in A_{p}^{+}$if and only if there exist $w_{1} \in A_{1}^{+}$and $w_{2} \in A_{1}^{-}$such that $w=w_{1}\left(w_{2}\right)^{1-p}$.

(3) Let $1<p<\infty$. Then $w \in A_{p}^{+}$if and only if $w^{1-p^{\prime}} \in A_{p^{\prime}}^{-}$, where $\frac{1}{p}+\frac{1}{p^{\prime}}=1$.

(4) Let $1<p<\infty$ and $w \in A_{p}^{+}$. Then $A_{p}^{+}\left(\delta^{\lambda}(w)\right)=A_{p}^{+}(w)$, where $\delta^{\lambda}(w)(x)=w(\lambda x)$ for all $\lambda>0$.

In [30], the authors obtained some weighted norm estimates for $M_{b}^{k,+}$.

Lemma 2.2 The following conditions are equivalent: 
(1) $M_{b}^{k,+}$ is bounded on $L^{p}(w)$ for every $1<p<\infty$ with $w \in A_{p}^{+}$.

(2) $M_{b}^{k,+}$ is bounded on $L^{p}(d x)$ for some $1<p<\infty$.

(3) $b \in B M O(\mathbb{R})$.

\section{Lemma 2.3 [33]}

(1) Let $1<p<\infty$, and let $b \in B M O(\mathbb{R})$. Then there exists $\lambda>0$ such that $e^{\lambda b} \in A_{p}^{+}$.

(2) Let $1<p<\infty$ and $\lambda>0$. Then there exists $\eta=\eta(\lambda, p)>0$ such that for $b \in B M O(\mathbb{R})$ and $\|b\|_{B M O(\mathbb{R})}<\eta$, we have $e^{\lambda b} \in A_{p}^{+}$.

To prove Theorem 1.1, we still need a celebrated interpolation theorem of operators with change of measures.

Lemma 2.4 [34] Suppose that $u_{0}, v_{0}, u_{1}, v_{1}$ are positive weight functions, and $1<p_{0}, p_{1}<$ $\infty$. Assume that a sublinear operator $S$ satisfies

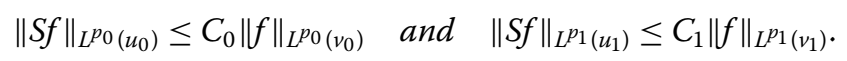

Then

$$
\|S f\|_{L^{p}(u)} \leq C\|f\|_{L^{p}(v)}
$$

for any $0<\theta<1$ and $1 / p=\theta / p_{0}+(1-\theta) / p_{1}$, where $u=u_{0}^{p \theta / p_{0}} u_{1}^{p(1-\theta) / p_{1}}, v=v_{0}^{p \theta / p_{0}} v_{1}^{p(1-\theta) / p_{1}}$, and $C \leq C_{0}^{\theta} C_{1}^{1-\theta}$.

\section{Proofs of the main results}

The proof of Theorem 1.1 is a by-product of the following two lemmas.

Lemma 3.1 Let $p, K, k, w$, and $b$ be as in Theorem 1.1. Then the operator

$$
T_{b, 0}^{k,+} f(x)=\text { p.v. } \int_{x}^{x+1} e^{i P(x, y)} K(x-y) f(y)(b(x)-b(y))^{k} d y
$$

is bounded on $L^{p}(w)$.

Lemma 3.2 Let $p, K, k, w$, and $b$ be as in Theorem 1.1. Then the operator

$$
T_{b, \infty}^{k,+} f(x)=\int_{x+1}^{\infty} e^{i P(x, y)} K(x-y) f(y)(b(x)-b(y))^{k} d y
$$

is bounded on $L^{p}(w)$.

\subsection{Proof of Lemma 3.1}

Lemma 3.1 can be proved by induction on $k$. For $k=0$, by [16], Theorem 1.3, Lemma 3.3, we have the boundedness of the operator

$$
T_{b, 0}^{0,+} f(x)=: T_{0}^{+} f(x)=\text { p.v. } \int_{x}^{x+1} e^{i P(x, y)} K(x-y) f(y) d y
$$


on $L^{p}(w)$. We now assume that Lemma 3.1 holds for $k-1$, that is, for any $f \in L^{p}(w)$ with $w \in A_{p}^{+}$, we have

$$
\left\|T_{b, 0}^{k-1,+} f\right\|_{L^{p}(w)} \leq C\|f\|_{L^{p}(w)}
$$

Next, we claim that

$$
\left\|T_{b, 0}^{k,+} f\right\|_{L^{p}(w)} \leq C\|f\|_{L^{p}(w)}
$$

In fact, by Lemma 2.1 there exists $\varepsilon>0$ such that, for any $f \in L^{p}\left(w^{1+\varepsilon}\right)$,

$$
\left\|T_{b, 0}^{k-1,+} f\right\|_{L^{p}\left(w^{1+\varepsilon}\right)} \leq C\|f\|_{L^{p}\left(w^{1+\varepsilon}\right)}
$$

By Lemma 2.3 with $\lambda=\frac{p(1+\varepsilon)}{\varepsilon}$, there is $\eta>0$ such that

$$
e^{\frac{p b(1+\varepsilon)}{\varepsilon}} \in A_{p}^{+} \quad \text { whenever }\|b\|_{B M O(\mathbb{R})}<\eta .
$$

On the other hand, the fact that for every $\theta \in[0,2 \pi], b \cos \theta \in B M O(\mathbb{R})$ and

$$
\|b \cos \theta\|_{B M O(\mathbb{R})} \leq\|b\|_{B M O(\mathbb{R})}<\eta
$$

shows that $e^{g(p, b, \varepsilon, \theta)} \in A_{p}^{+}$with $g(p, b, \varepsilon, \theta):=\frac{p b(1+\varepsilon) \cos \theta}{\varepsilon}$. Therefore, we can conclude from (3.1) that for every $\theta \in[0,2 \pi]$,

$$
\left\|T_{b, 0}^{k-1,+} f\right\|_{L^{p}(g(p, b, \varepsilon, \theta))} \leq C\|f\|_{L^{p}(g(p, b, \varepsilon, \theta))}
$$

where $C$ depends on $p, b$, and $w$ but not on $k$ and $\theta$. Applying Lemma 2.4 to (3.2) and (3.3), we have

$$
\left\|T_{b, 0}^{k-1,+} f\right\|_{L^{p}\left(w e^{p b \cos \theta}\right)} \leq C\|f\|_{L^{p}(w e p b \cos \theta)} \text { for every } \theta \in[0,2 \pi]
$$

where $C$ and $\delta>0$ are independent of $k$ and $\theta$. Moreover, if we set $g_{\theta}(x)=f(x) e^{-b(x) e^{i \theta}}$, then $g_{\theta} \in L^{p}\left(w e^{p b \cos \theta}\right)$ and

$$
\left\|g_{\theta}\right\|_{L^{p}(w e p b \cos \theta)}=\|f\|_{L^{p}(w)} .
$$

For simplicity, we denote

$$
K_{k-1}(x, y)=e^{i P(x, y)} K(x-y)(b(x)-b(y))^{k-1} \chi_{0<y-x<1}(x-y) .
$$

Now, for $z \in \mathbb{C}$ (here $z$ is not of the form $z=x+i y$, with $x, y$ variables of $P(x, y)), g(z)=$ $e^{z(b(x)-b(y))}$ is analytic on $\mathbb{C}$. Thus, by the Cauchy integral formula we get

$$
b(x)-b(y)=g^{\prime}(0)=\frac{1}{2 \pi i} \int_{|z|=1} \frac{g(z)}{|z|^{2}} d z=\frac{1}{2 \pi} \int_{0}^{2 \pi} e^{i \theta[b(x)-b(y)]} e^{-i \theta} d \theta .
$$


Then

$$
T_{b, 0}^{k,+} f(x)=\int_{x}^{x+1} K_{k-1}(x, y)(b(x)-b(y)) f(y) d y=\frac{1}{2 \pi} \int_{0}^{2 \pi} T_{b, 0}^{k-1,+}\left(g_{\theta}\right)(x) e^{e^{i \theta} b(x)} e^{-i \theta} d \theta .
$$

This, combined with (3.1), (3.4), and Minkowski's inequality, gives

$$
\begin{aligned}
\left\|T_{b, 0}^{k,+} f\right\|_{L^{p}(w)} & \leq \frac{1}{2 \pi} \int_{0}^{2 \pi}\left\|T_{b, 0}^{k-1,+}\left(g_{\theta}\right)\right\|_{L^{p}(w e p b \cos \theta)} d \theta \\
& \leq C \frac{1}{2 \pi} \int_{0}^{2 \pi}\left\|g_{\theta}\right\|_{L^{p}\left(w e e^{p \cos \theta}\right)} d \theta \\
& =C\|f\|_{L^{p}(w)}
\end{aligned}
$$

which shows Lemma 3.1 .

\subsection{Proof of Lemma 3.2}

We can proceed analogously to the proof of Lemma 3.2 by induction as in Lemma 3.1. Write

$$
T_{b, \infty}^{k,+} f(x)=\sum_{j=1}^{\infty} T_{b, j}^{k,+} f(x)=\sum_{j=1}^{\infty} \int_{x+2^{j-1}}^{x+22^{j}} e^{i P(x, y)} K(x-y)(b(x)-b(y))^{k} f(y) .
$$

We next claim that, for some $\delta>0$,

$$
\left\|T_{b, j}^{k,+} f\right\|_{L^{p}(w)} \leq 2^{-\delta j}\|f\|_{L^{p}(w)}
$$

Indeed, when $k=0$, we have

$$
\left\|T_{b, j}^{0,+} f\right\|_{L^{p}}=\left\|T_{j}^{+} f\right\|_{L^{p}} \leq C 2^{-\eta j}\|f\|_{L^{p}}
$$

following arguments similar to those in [16], p.157, where $C$ depends only on the total degree of $P$ and $\eta>0$. On the other hand, it is easy to check that

$$
\left|T_{b, j}^{0,+} f(x)\right|=\left|T_{j}^{+} f(x)\right| \leq C \int_{x+2^{j-1}}^{x+2^{j}} \frac{|f(y)|}{|x-y|} d y \leq C M^{+}(f)(x),
$$

where $C$ is independent of $j$. Then it follows from Lemma 2.1 and Lemma 2.2 that there exists $\varepsilon>0$ such that $w^{1+\varepsilon} \in A_{p}^{+}$and

$$
\left\|T_{b, j}^{0,+} f\right\|_{L^{p}\left(w^{1+\varepsilon}\right)} \leq C\|f\|_{L^{p}\left(w^{1+\varepsilon}\right)}
$$

where $C$ is independent of $j$. Applying Lemma 2.4 to inequalities (3.6) and (3.7), it follows that

$$
\left\|T_{b, j}^{0,+} f\right\|_{L^{p}(w)} \leq C 2^{-\eta_{1} j}\|f\|_{L^{p}(w)}
$$

where $\eta_{1}>0$ is independent of $j$ and $f$. Inequality (3.8) implies that (3.5) holds for $k=0$. 
We now assume that (3.5) holds for $k-1$, that is, for any $f \in L^{p}(w)$ with $w \in A_{p}^{+}$,

$$
\left\|T_{b, j}^{k-1,+} f\right\|_{L^{p}(w)} \leq C 2^{-\eta_{k-1} j}\|f\|_{L^{p}(w)}
$$

By Lemma 2.1, given $w \in A_{p}^{+}$, there exists $\varepsilon>0$ such that $w^{1+\varepsilon} \in A_{p}^{+}$. Then for any $f \in$ $L^{p}\left(w^{1+\varepsilon}\right)$, we have

$$
\left\|T_{b, j}^{k-1,+} f\right\|_{L^{p}\left(w^{1+\varepsilon}\right)} \leq C 2^{-\eta_{k-1}^{\prime}}\|f\|_{L^{p}\left(w^{1+\varepsilon}\right)}
$$

Taking $\lambda=\frac{p(1+\varepsilon)}{\varepsilon}$, by Lemma 2.3 there exists $\eta>0$ such that

$$
e^{\frac{p b(1+\varepsilon)}{\varepsilon}} \in A_{p}^{+} \quad \text { whenever }\|b\|_{B M O(\mathbb{R})}<\eta .
$$

On the other hand, for every $\theta \in[0,2 \pi], b \cos \theta \in B M O(\mathbb{R})$ and

$$
\|b \cos \theta\|_{B M O(\mathbb{R})} \leq\|b\|_{B M O(\mathbb{R})}<\eta .
$$

Thus, $e^{g(p, b, \varepsilon, \theta)} \in A_{p}^{+}$for $g(p, b, \varepsilon, \theta):=\frac{p b(1+\varepsilon) \cos \theta}{\varepsilon}$. Hence, by inequality (3.9) we have

$$
\left\|T_{b, j}^{k-1,+} f\right\|_{L^{p}\left(e^{g(p, b, \varepsilon, \theta)}\right)} \leq C 2^{-\eta_{k-1}^{\prime \prime} j}\|f\|_{L^{p}\left(e^{g(p, b, \varepsilon, \theta)}\right)},
$$

where $C$ and $\eta_{k-1}^{\prime \prime}$ depend on $p, b$, and $w$ but not on $j, k$, and $\theta$.

Applying Lemma 2.4 to inequalities (3.10) and (3.11), we have

$$
\left\|T_{b, j}^{k-1,+} f\right\|_{L^{p}\left(w e^{p b \cos \theta}\right)} \leq C 2^{-\eta j}\|f\|_{L^{p}\left(w e^{p b \cos \theta}\right)},
$$

where $C$ and $\delta>0$ are independent of $j, k$, and $\theta$. Setting $g_{\theta}(x)=f(x) e^{-b(x) e^{i \theta}}$, it is easy to check that for $\theta \in[0,2 \pi]$, we have $g_{\theta} \in L^{p}\left(w e^{p b \cos \theta}\right)$ and

$$
\left\|g_{\theta}\right\|_{L^{p}\left(w e^{p b \cos \theta}\right)}=\|f\|_{L^{p}(w)} .
$$

\section{Denote by}

$$
K_{k-1}(x, y)=e^{i P(x, y)} K(x-y)(b(x)-b(y))^{k-1} \chi_{2^{j-1}<y-x<2^{j}}(x-y) .
$$

Then, for $z \in \mathbb{C}, g(z)=e^{z(b(x)-b(y))}$ is analytic on $\mathbb{C}$, which shows

$$
b(x)-b(y)=g^{\prime}(0)=\frac{1}{2 \pi i} \int_{|z|=1} \frac{g(z)}{|z|^{2}} d z=\frac{1}{2 \pi} \int_{0}^{2 \pi} e^{e^{i \theta}[b(x)-b(y)]} e^{-i \theta} d \theta .
$$

Thus,

$$
\begin{aligned}
T_{b, j}^{k,+} f(x) & =\int_{x+2^{j-1}}^{x+2^{j}} K_{k-1}(x, y)(b(x)-b(y)) f(y) d y \\
& =\frac{1}{2 \pi} \int_{0}^{2 \pi} T_{b, j}^{k-1,+}\left(g_{\theta}\right)(x) e^{e^{i \theta} b(x)} e^{-i \theta} d \theta .
\end{aligned}
$$


Finally, combining (3.9), (3.12), and the Minkowski inequality, we get the following estimate:

$$
\begin{aligned}
\left\|T_{b, j}^{k,+} f\right\|_{L^{p}(w)} & \leq \frac{1}{2 \pi} \int_{0}^{2 \pi}\left\|T_{b, j}^{k-1,+}\left(g_{\theta}\right)\right\|_{L^{p}(w e p b \cos \theta)} d \theta \\
& \leq C \frac{1}{2 \pi} \int_{0}^{2 \pi} 2^{-\delta j}\left\|g_{\theta}\right\|_{L^{p}\left(w e^{p b \cos \theta}\right)} d \theta \\
& =C 2^{-\delta j}\|f\|_{L^{p}(w)} .
\end{aligned}
$$

We thus complete the proof of Lemma 3.2 by the following observation:

$$
\left\|T_{b, \infty}^{k,+} f\right\|_{L^{p}(w)} \leq \sum_{j=1}^{\infty}\left\|T_{b, j}^{k,+} f\right\|_{L^{p}(w)} \leq C\|f\|_{L^{p}(w)}
$$

\subsection{Proof of Theorem 1.2}

For any $\lambda \in \mathbb{R}$, if a nontrivial polynomial $P(x, y)$ satisfies

$$
\begin{aligned}
P(x, y) & =\sum_{\alpha, \beta} a_{\alpha, \beta}(x-\lambda)^{\alpha}(y-\lambda)^{\beta}+R_{1}(x, \lambda)+R_{2}(y, \lambda) \\
& :=P(x-\lambda, y-\lambda)+R_{1}(x, \lambda)+R_{2}(y, \lambda),
\end{aligned}
$$

where $R_{1}$ and $R_{2}$ are real polynomials, then by Theorem 1.1, $T_{b}^{k,+}$ is bounded on $L^{p}(w)$. Rewrite the kernel $K$ as

$$
K(x-y)=K_{0}(x-y)+K_{\infty}(x-y):=K(x-y) \chi_{\{|x-y|<1\}}(y)+K(x-y) \chi_{\{|x-y|>1\}}(y)
$$

and consider the corresponding splitting

$$
\begin{aligned}
T_{b}^{k,+} f(x)= & T_{b, 0}^{k,+} f(x)+T_{b, \infty}^{k,+} f(x) \\
=: & \int_{x}^{\infty} e^{i P(x, y)} K_{0}(x-y)(b(x)-b(y))^{k} f(y) d y \\
& +\int_{x}^{\infty} e^{i P(x, y)} K_{\infty}(x-y)(b(x)-b(y))^{k} f(y) d y .
\end{aligned}
$$

Since both $T_{b, \infty}^{k,+}$ and $T_{b, 0}^{k,+}$ are bounded on $L^{p}(w)$, it follows that, for any $h \in \mathbb{R}$,

$$
\left(\int_{|x-h|<1}\left|T_{b, 0}^{k,+} f(x)\right|^{p} w(x) d x\right)^{\frac{1}{p}} \leq C\left(\int_{|y-h|<2}|f(y)|^{p} w(y) d y\right)^{\frac{1}{p}}
$$

where $C$ is independent of $h$ and $f$ (see also [35]).

For $h \in \mathbb{R}$, set

$$
\widetilde{T}_{b, 0}^{k,+} f(x)=e^{-i R_{1}(x, h)} \text { p.v. } \int_{x}^{x+1} e^{i P(x, y)} K_{k}(x, y) f(y) e^{-i P(x-h, y-h)} e^{-i R_{2}(y, h)} d y,
$$


where $K_{k}(x, y)=K(x-y)(b(x)-b(y))^{k}$. Then express $e^{-i P(x-h, y-h)}$ by the Taylor series

$$
\begin{aligned}
e^{-i P(x-h, y-h)} & =\sum_{m=0}^{\infty} \frac{(-i)^{m}}{m !}(P(x-h, y-h))^{m} \\
& =\sum_{m=0}^{\infty} \frac{(-i)^{m}}{m !}\left(\sum_{\alpha, \beta} a_{\alpha, \beta}(x-h)^{\alpha}(y-h)^{\beta}\right)^{m} \\
& =\sum_{m=0}^{\infty} \frac{(-i)^{m}}{m !} \sum_{l} c_{m, l} b_{\mu, v, l}(x-t)^{\mu}(y-t)^{\nu},
\end{aligned}
$$

where $\mu:=\mu(\alpha, \beta, l)$ and $v:=v(\alpha, \beta, l)$ are multiindices. By (3.13), if we set $|x-h| \leq \xi<1$ and $|y-h| \leq \eta<2$, then we the inequalities

$$
\begin{aligned}
& \left(\int_{|x-h|<1}\left|\widetilde{T}_{b, 0}^{k,+} f(x)\right|^{p} w(x) d x\right)^{\frac{1}{p}} \\
& \quad \leq \sum_{m=0}^{\infty} \frac{\sum_{l}\left|c_{m, l} b_{\mu, v, l}\right|}{m !}\left(\int_{|x-h|<1}\left|T_{b, 0}^{k,+}\left[e^{-i R_{2}(\cdot, h)} f(\cdot)|\cdot-h|^{\nu}\right](x)\right|^{p} w(x) d x\right)^{\frac{1}{p}} \\
& \quad \leq \sum_{m=0}^{\infty} \frac{\sum_{l}\left|c_{m, l} b_{\mu, v, l}\right|}{m !}\left(\int_{|y-h|<2}\left|f(y)(y-h)^{v}\right|^{p} w(y) d y\right)^{\frac{1}{p}} \\
& \quad \leq C \sum_{m=0}^{\infty} \frac{\sum_{l}\left|c_{m, l} b_{\mu, v, l}\right|}{m !}\left(\int_{|y-h|<2}|f(y)|^{p} w(y) d y\right)^{\frac{1}{p}} \\
& \quad \leq C \sum_{m=0}^{\infty} \frac{\left(\sum_{\alpha, \beta}\left|a_{\alpha, \beta}\right| \xi^{\alpha} \eta^{\beta}\right)^{m}}{m !}\left(\int_{|y-h|<2}|f(y)|^{p} w(y) d y\right)^{\frac{1}{p}} \\
& \quad \leq C e^{\left(\sum_{\alpha, \beta}\left|a_{\alpha, \beta}\right|\right)}\left(\int_{|y-h|<2}|f(y)|^{p} w(y) d y\right)^{\frac{1}{p}} \\
& \quad \leq C\left(\int_{|y-h|<2}|f(y)|^{p} w(y) d y\right)^{\frac{1}{p}}
\end{aligned}
$$

for all $h \in \mathbb{R}$, which implies that $\widetilde{T}_{b, 0}^{k,+}$ is bounded on $L^{p}(w)$.

\section{Competing interests}

The authors declare that they have no competing interests.

\section{Authors' contributions}

All authors read and approved the final manuscript.

\section{Acknowledgements}

This work was partially supported by National Natural Science Foundation of China (Grant Nos. 11271175, 11301249) and the Applied Mathematics Enhancement Program of Linyi University (No. LYDX2013BS059). The authors thank the anonymous referees cordially for their valuable suggestions on this paper.

Received: 20 September 2015 Accepted: 18 February 2016 Published online: 03 March 2016

\section{References}

1. Ricci, F, Stein, E: Harmonic analysis on nilpotent groups and singular integrals I: oscillatory integrals. J. Funct. Anal. 73, 179-194 (1987)

2. Stein, E: Harmonic Analysis: Real-Variable Methods, Orthogonality, and Oscillatory Integrals. Princeton University Press, Princeton (1993) 
3. Lu, S, Zhang, Y: Weighted norm inequality of a class of oscillatory singular operators. Chin. Sci. Bull. 37, 9-13 (1992)

4. Lu, S, Zhang, Y: Criterion on $L^{p}$-boundedness for a class of oscillatory singular integrals with rough kernels. Rev. Mat. Iberoam. 8, 201-219 (1992)

5. Chanillo, S, Christ, M: Weak $(1,1)$ bounds for oscillatory integrals. Duke Math. J. 55, 141-155 (1987)

6. Grafakos, L: Classical and Modern Fourier Analysis. Pearson Education, Upper Saddle River (2004)

7. Hu, Y, Pan, Y: Boundedness of oscillatory singular integrals on Hardy spaces. Ark. Mat. 30, 311-320 (1992)

8. Lu, S: Multilinear oscillatory integrals with Calderón-Zygmund kernel. Sci. China Ser. A 42, 1039-1046 (1999)

9. Pan, Y: Hardy spaces and oscillatory integral operators. Rev. Mat. Iberoam. 7, 55-64 (1991)

10. Sato, S: Weighted weak type $(1,1)$ estimates for oscillatory integrals. Stud. Math. 141, 1-24 (2000)

11. Sawyer, E: Weighted inequalities for the one-sided Hardy-Littlewood maximal function. Trans. Am. Math. Soc. 297, 53-61 (1986)

12. Martín-Reyes, F, Pirk, L, de la Torre, A: $A_{\infty}^{+}$Condition. Can. J. Math. 45, 1231-1244 (1993)

13. Aimar, H, Forzani, L, Martín-Reyes, F: On weighted inequalities for one-sided singular integrals. Proc. Am. Math. Soc. $125,2057-2064$ (1997)

14. Fu, Z, Lu, S, Pan, Y, Shi, S: Boundedness of one-sided oscillatory integral operators on weighted Lebesgue spaces. Abstr. Appl. Anal. 2014, Article ID 291397 (2014)

15. Fu, Z, Lu, S, Sato, S, Shi, S: On weighted weak type norm inequalities for one-sided oscillatory singular integrals. Stud. Math. 207, 137-151 (2011)

16. Fu, Z, Lu, S, Pan, Y, Shi, S: Some one-sided estimates for oscillatory singular integrals. Nonlinear Anal. 108, 144-160 (2014)

17. Coifman, R, Rochbery, R, Weiss, G: Factorization theorems for Hardy spaces in several variables. Ann. Math. 103, 611-635 (1976)

18. Janson, S: Mean oscillation and commutators of singular integral operators. Ark. Mat. 16, 263-270 (1978)

19. Paluszynski, M: Characterization of the Besov spaces via the commutator operator of Coifman, Rochberg and Weiss. Indiana Univ. Math. J. 44, 1-17 (1995)

20. Chiarenza, F, Frasca, M, Longo, P: W2,P-Solvability of the Dirichlet problem for nondivergence elliptic equations with VMO coefficients. Trans. Am. Math. Soc. 336, 841-853 (1993)

21. Fazio, G, Ragusa, M: Interior estimates in Morrey spaces for strongly solutions to nondivergence form equations with discontinuous coefficients. J. Funct. Anal. 112, 241-256 (1993)

22. Alvarez, J, Bagby, R, Kurtz, D, Perez, C: Weighted estimates for commutators of linear operators. Stud. Math. 104, 195-209 (1993)

23. Ding, Y, Lu, S: Weighted $L^{p}$-boundedness for higher order commutators of oscillatory singular integrals. Tohoku Math J. 48, 437-449 (1996)

24. Lu, S, Ding, Y, Yan, D: Singular Integrals and Related Topics. World Scientific Publishing, Singapore (2007)

25. Lu, S, Wu, H: Oscillatory singular integrals and commutators with rough kernels. Ann. Sci. Math. Qué. 27, 47-66 (2003)

26. Lu, $S, Y$ Yan, $D: L^{p}$-Boundedness of multilinear oscillatory singular integrals with Calderón-Zygmund kernel. Sci. China Ser. A 45, 196-213 (2002)

27. $\mathrm{Wu}, \mathrm{H}$ : Boundedness of higher order commutators of oscillatory singular integrals with rough kernels. Stud. Math. $167,29-43(2005)$

28. Yan, D: Some problems on multilinear integral operators and oscillatory singular integral operators. Ph.D. Dissertation, Beijing Normal University (2001)

29. Lorente, M, Riveros, M: Weighted inequalities for commutators of one-sided singular integrals. Comment. Math. Univ. Carol. 43, 83-101 (2002)

30. Lorente, M, Riveros, M: Weights for commutators of one-sided discrete square function, the Weyl fractional integral and other one-sided operators. Proc. R. Soc. Edinb. A 135, 845-862 (2005)

31. Shi, S, Fu, Z, Lu, S: Weighted estimates for commutators of one-sided oscillatory operators. Front. Math. China 6 , 507-516 (2011)

32. Riveros, M, de la Torre, A: On the best ranges for $A_{p}^{+}$and $R H_{r}^{+}$. Czechoslov. Math. J. 126, $285-301$ (2001)

33. Martín-Reyes, F, de la Torre, A: One-sided BMO spaces. J. Lond. Math. Soc. 49, 529-542 (1994)

34. Stein, E, Weiss, G: Interpolation of operators with change of measures. Trans. Am. Math. Soc. 87, 159-172 (1958)

35. Jing, Y, Lu, S: Oscillatory singular integrals with rough kernel. In: Harmonic Analysis in China. Math. Appl., vol. 327, pp. 135-145. Kluwer Academic, Dordrecht (1995)

\section{Submit your manuscript to a SpringerOpen ${ }^{\circ}$ journal and benefit from:}

- Convenient online submission

Rigorous peer review

- Immediate publication on acceptance

- Open access: articles freely available online

- High visibility within the field

- Retaining the copyright to your article 